\title{
Noninvasive Ventilation in Patients with Severe Acute Respiratory Syndrome
}

\author{
David S.C. Hui
}

Keywords

$\mathrm{NIV} \bullet \mathrm{SARS} \bullet$ Emerging respiratory infections

\subsection{Introduction}

Severe acute respiratory syndrome first emerged in Guangdong, China in November 2002 and then spread rapidly to many countries through Hong Kong in 2003 [1-4]. A 64-year-old physician from southern China, who had visited Hong Kong on February 21, 2003 and died 10 days later of severe pneumonia, is believed to have been the source of infection causing subsequent outbreaks of severe acute respiratory syndrome (SARS) in Hong Kong, Vietnam, Singapore, and Canada [1-4]. By the end of the epidemic in July 2003, there had been 8,096 cases reported in 29 countries and regions, with a mortality incidence of 774 (9.6\%) [5]. Among the 8,096 cases, 1,706 were health care workers (HCWs). A novel coronavirus (CoV) was responsible for SARS [6]. Bats are likely the natural reservoirs of SARS-like $\mathrm{CoV}[7,8]$.

The clinical course of SARS generally follows a typical pattern [9]. Phase 1 (viral replication) is associated with an increasing viral load during the first week of the illness and is clinically characterized by fever, myalgia, and other systemic symptoms that generally diminish after a few days. Phase 2 (immunopathological injury) is characterized by recurrence of fever, hypoxemia, and radiological progression of pneumonia with falls in viral load during the second week of illness.

\footnotetext{
D.S.C. Hui, MD

Department of Medicine \& Therapeutics, The Chinese University of Hong Kong, Prince of Wales Hospital, 30-32 Ngan Shing St., Shatin, N.T., Hong Kong

Stanley Ho Center for Emerging Infectious Diseases,

The Chinese University of Hong Kong, Shatin, N.T., Hong Kong e-mail: dschui@cuhk.edu.hk
} 
The high morbidity associated with SARS was highlighted by the observation that even when only $12 \%$ of the total lung field is involved by consolidation on chest radiographs, $50 \%$ of patients require supplemental oxygen to maintain satisfactory oxygenation above $90 \%$ [10]. Peiris et al. [9] showed peaking of the nasopharyngeal viral load on day 10 of illness followed by a progressive decrease in rates of viral shedding from the nasopharynx, in stool, and in urine from day 10 to day 21 after symptom onset in 20 patients who had serial measurements with reverse transcription-polymerase chain reaction. Thus, clinical worsening of patients with SARS during phase 2 (second week of illness) is most likely the result of immunemediated lung injury due to an overexuberant host response rather than uncontrolled viral replication [9].

SARS spreads mainly by close person-to-person contact via droplet transmission or fomite [11]. During the global outbreak of SARS, about $20 \%$ of patients progressed into acute respiratory distress syndrome (ARDS), necessitating invasive ventilatory support while reaching a very high viral load at the nasopharynx with the peak on day 10 of illness [9]. Thus, HCWs were particularly prone to infection while caring for patients at a close distance [1, 9, 12]. These data emphasize the need for adequate respiratory protection in addition to strict contact and droplet precautions when managing patients with pneumonia due to highly infectious diseases.

\subsection{Studies Reporting Safe Application of NIV to Patients with SARS}

Several uncontrolled studies have shown that single-circuit noninvasive ventilation (NIV) can be life-saving for patients in respiratory failure due to SARS infection [13-15]. Among 120 patients meeting clinical criteria for SARS who were in a hospital for infectious diseases in Beijing, 30 (25\%) had developed acute respiratory failure $(\mathrm{ARF})$ at $10.7 \pm 3.8$ days after the onset of SARS. Among these 30 patients, $16(53 \%)$ exhibited hypercapnia $\left(\mathrm{PaCO}_{2}>45 \mathrm{mmHg}\right)$, and 10 hypercapnic events occurred within 1 week of admission. NIV was instituted in 28 patients, with 1 patient intolerant of it. In the remaining 27 patients with SARS, NIV was initiated $1.2 \pm 1.6$ days after ARF onset. An hour of NIV therapy led to significant improvement in $\mathrm{PaO}_{2} / \mathrm{FiO}_{2}$ and a reduced respiratory rate $(p<0.01)$. Endotracheal intubation was required in one-third of the patients $(9 / 27)$ despite a favorable response to NIV initially. Remarkable pulmonary barotrauma was noted in 7 of the 120 patients $(5.8 \%)$ and in 6 of those $(22 \%)$ on NIV. The overall fatality rate at 13 weeks was $6.7 \%(8 / 120)$. It was higher $(26.7 \%)$ among those needing NIV. None of the HCWs contracted SARS. The authors concluded that NIV is a feasible, appropriate treatment for ARF due to SARS infection [13].

In another study, NIV was applied via oronasal mask to 20 SARS patients without chronic obstructive pulmonary disease (COPD) who had developed severe hypoxemic respiratory failure in a hospital in Hong Kong with efficient room air exchange (through timely installation of powerful exhaust fans to provide 8-12 air 
changes per hour), stringent infection control measures, full personal protective equipment (PPE), and addition of a viral/bacterial filter to the exhalation port of the NIV device. The mean age of the patients was 51.4 years. The mean Acute Physiology and Chronic Health Evaluation (APACHE) II score was 5.35. SARS CoV serology was positive in 19 of 20 patients (95\%). NIV was started at a mean of 9.6 days from symptom onset. The mean duration of NIV usage was $84.3 \mathrm{~h}$. Endotracheal intubation was avoided in 14 patients $(70 \%)$, in whom the ICU stay was shorter than those who required intubation (3.1 vs. 21.3 days, $p<0.001$ ), and the chest radiography score within $24 \mathrm{~h}$ of NIV was lower (15.1 vs. $22.5, p=0.005$ ) compared to the intubated patients. Intubation avoidance was predicted by a marked reduction in respiratory rate ( 9.2 breaths per $\mathrm{min}$ ) and supplemental oxygen requirement $(3.1 \mathrm{~L} / \mathrm{min})$ within $24 \mathrm{~h}$ of NIV. There were no clinical infections among the $105 \mathrm{HCWs}$ caring for the 20 patients receiving NIV, and $102 \mathrm{HCWs}$ who had consented to blood serology testing were all negative for SARS CoV. NIV appeared effective in the treatment of ARF in the patients with SARS, and its use was safe for HCWs in this single-center study [14].

A retrospective analysis was conducted on all patients with respiratory failure identified from the Hong Kong Hospital Authority SARS Database. Intubation rate, mortality, and secondary outcome of a hospital utilizing NIV under standard infection control conditions (the same NIV hospital as described above [14]) were compared to 13 other hospitals using only invasive mechanical ventilation (IMV hospitals) [15]. The two hospital groups had comparable demographics and clinical profiles, but patients at the NIV hospital $(n=42)$ had higher lactate dehydrogenase levels and worse radiographic scores on admission. Compared to the IMV hospitals $(n=451)$, the NIV hospital had lower adjusted odds ratios (OR) for intubation [0.36, $95 \%$ confidence interval (CI) $0.164-0.791, p=0.011]$ and death $(0.235,95 \% \mathrm{CI}$ $0.077-0.716, p=0.011)$. There was no clinical transmission of SARS among HCWs caused by the use of NIV. Compared to IMV, NIV as the initial ventilatory support for ARF in the presence of SARS appeared to be safe. Also, it is associated with a reduced need for IMV and low mortality in this study [15].

\subsection{Studies Reporting Increased Risk of Transmission of SARS to HCWs via NIV}

A retrospective study by Xiao et al. [16] described NIV exposure as a risk factor associated with clinical SARS infection in two HCWs in Guangzhou, China. Other risk factors included involvement in patient resuscitation and IMV [16].

The relative risk of developing SARS was 13-fold for HCWs in Toronto who were involved in intubating SARS patients versus those who were not. In contrast, NIV was not associated with a statistically significant risk for the HCWs $(1 / 6$ exposed HCWs vs. 2/28 nonexposed, risk ratio 2.33, $p=0.5$ ) [17]. This was probably because tracheal suctioning was not generally performed for patients ventilated with NIV, and the study sample size was small [17]. In a subsequent retrospective multi-center cohort study of more than $600 \mathrm{HCW}$ sho were involved in managing 
SARS patients in Toronto, their presence in the room during fiberoptic intubation (OR 2.79, $p=0.004$ ) or electrocardiography (OR 3.52, $p=0.002)$, unprotected eye contact with secretions ( $\mathrm{OR}=7.34, p=0.001)$, patient APACHE II score $\geq 20$ (OR 17.05, $p=0.009)$, and patient $\mathrm{PaO}_{2} / \mathrm{FiO}_{2} \leq 59(\mathrm{OR} 8.65, p=0.001)$ were associated with increased risk of transmission of SARS CoV [18].

In a large case-control study involving 124 medical wards in 26 hospitals in Guangzhou and Hong Kong, NIV was identified as an independent risk factor for super-spreading nosocomial outbreaks of SARS (OR 11.82, $95 \%$ CI 1.97-70.80, $p=0.007$ ) [19].

A systematic review of five case-control and five retrospective cohort studies related to SARS identified four procedures that were associated with an increased risk of transmission of SARS to HCWs [20].

- Tracheal intubation $[n=4$, cohort: OR 6.6, $95 \%$ CI 2.3-18.9; and $n=4$ casecontrol: OR 6.6, $95 \%$ CI 4.1-10.6]

- NIV [n=2, cohort: OR 3.1, $95 \%$ CI 1.4-6.8]

- Tracheotomy [ $n=1$, case-control: OR 4.2, $95 \%$ CI 1.5-11.5]

- Manual ventilation before intubation [ $n=1$, cohort: OR 2.8, $95 \%$ CI 1.3-6.4]

In addition, there was an influenza outbreak investigation conducted with computer fluid dynamics analysis. It described application of NIV to a patient hospitalized with hypercapnic respiratory failure due to acute exacerbation of COPD by influenza $\mathrm{A}(\mathrm{H} 3 \mathrm{~N} 2$ virus). The patient was in a general medical ward with imbalanced airflow related to different high-efficiency particulate air (HEPA) filter settings in the ward that appeared to have converted droplets that were subjected to airborne transmission. It resulted in nosocomial infection due to the same influenza $\mathrm{A}(\mathrm{H} 3 \mathrm{~N} 2)$ virus seen in the first patient, affecting several other patients in the adjacent bay on the same ward [21].

\subsection{Technical and Infection Control Considerations}

Noninvasive ventilation should be commenced under strict infection control measures, as recommended in Table 13.1 for patients with SARS and other emerging respiratory infections. It is started in patients in whom nasal oxygen $>5 \mathrm{~L} / \mathrm{min}$ fails to maintain the target $\mathrm{SpO}_{2}(93-96 \%)$. Inspiratory positive airway pressure (IPAP) is adjusted to achieve a respiratory rate of $<25$ breaths per minute and exhaled tidal volumes $>6 \mathrm{~mL} / \mathrm{kg}$. Expiratory positive airway pressure (EPAP) is adjusted to achieve target oxygenation with minimum carbon dioxide rebreathing. The criteria for switching to intubation include intolerance to NIV, patient fatigue, or when supplemental oxygen at $12 \mathrm{~L} / \mathrm{min}$ fails to maintain at least $93 \% \mathrm{SpO}_{2}$ while on NIV [15].

All HCWs should take precautions when managing patients with communityacquired pneumonia (CAP) of unknown etiology that is complicated by respiratory failure. Experimental studies based on a sophisticated human patient simulator and laser visualization technique have shown that the maximum exhaled air particle dispersion distance from patients receiving NIV via the ResMed Ultra Mirage mask was about $0.5 \mathrm{~m}$ along the exhalation port [22]. The same research group 
Table 13.1 Infection control precautions in the ICU for management of SARS [15, 26]

\section{Staff education}

(a) Limit opportunities for exposure: limit aerosol generating procedures and limit number of HCWs present.

(b) Effective use of time during patient contact.

(c) How to "gown up" and "gown down" without contamination.

(d) Emphasis on importance of vigilance and adherence to all infection control measures in addition to monitoring own health.

Personal protective equipment (PPE)

(a) N95 respirator for airborne and surgical mask for droplet precautions.

(b) Contact precautions: Disposable gloves, gown, and cap.

(c) Eye protection with nonreusable goggles and face shield.

(d) Powered air purification respirators for use when performing high-risk procedures.

(e) No pens, paper, other personal items, or medical records allowed into or removed from the room.

(f) Immediate removal of grossly contaminated PPE and showering in nearby facility.

Environmentlequipment

(a) Conform to $\mathrm{CDC}$ recommendation for environmental control of tuberculosis: minimum 6 air changes per hour (ACHs). Where feasible, increase to $12 \mathrm{ACHs}$ or recirculate air through HEPA filter.

(b) Preferred: negative pressure isolation rooms with antechambers, with doors closed at all times.

(c) Equipment not be shared among patients.

(d) Alcohol-based hand and equipment disinfectants.

(e) Gloves, gowns, masks, and disposal units readily available.

(f) Careful, frequent cleaning of surfaces with disposable cloths and alcohol-based detergents.

(g) Use of video camera equipment or windows to monitor patients.

\section{Transport}

Avoid patient transport where possible. Balance risks and benefits of investigations that necessitate patient transport.

Special precautions for ICU

(a) Viral/bacterial filter placed in expiratory port of bag-valve mask.

(b) Two filters per ventilator: between expiratory port and the ventilator; another on the exhalation outlet of the ventilator.

(c) Closed system in-line suctioning of endotracheal/tracheostomy tubes.

(d) Heat and moisture exchanger (HME) preferred to heated humidifier. Careful handling of contaminated HME.

(e) Scavenger system for exhalation port of ventilator. Optional if negative pressure with high air exchange $(>12 / \mathrm{h})$ is achieved.

demonstrated that the maximum exhaled air dispersion distance from the Respironics ComfortFull 2 mask was about $1 \mathrm{~m}$ at a predictable direction from the exhalation diffuser perpendicular to the patient. Leakage though the Respironics Image 3 mask, connected to the whisper swivel exhalation port, was much more extensive and diffuse even at a low IPAP of $10 \mathrm{cmH}_{2} \mathrm{O}$ [23]. The whisper swivel is an efficient exhalation device to prevent carbon dioxide rebreathing, but it is not advisable to use such an exhalation port when managing patients with highly infectious conditions such as SARS for fear of causing major nosocomial infection. It is also important to avoid the use of high IPAP, which could lead to wider distribution of 
Table 13.2 Recommendations by the European Respiratory Society and European Society of Intensive Care Medicine for use of NIV during the pandemic 2009 influenza A(H1N1) infection [27]

(a) Prudent isolation of the patient coupled to protective measures for HCWs and other patients are the keys to limiting disease transmission.

(b) Use double-circuit tubes (or special filters for nonrebreathing devices).

(c) Minimize leaks.

(d) Use full-face masks or helmets.

(e) Avoid heated humidification.

(f) Protect hospital personnel with standard measures (i.e., wearing gloves, washing hands, use of masks, "negative pressure" rooms).

(g) Discard all masks, circuits, filters, and headsets immediately and safely after use according to routine infection control procedures. Routine exterior cleaning of ventilators and replacement of external filters should be sufficient to stop the spread of infection if ventilators are used on other NIV patients with H1NI. Complete decontamination may be considered before ventilators are used for patients without H1N1.

exhaled air and substantial room contamination [23]. These data have important clinical implications regarding the prevention of any future nosocomial outbreaks of SARS and other highly infectious conditions such as pandemic influenza.

Noninvasive ventilation should be applied in patients with severe CAP only if there is adequate protection for HCWs because of the potential risk of transmission. The organisms can spread via either deliberate or accidental mask interface leakage and flow compensation causing dispersion of contaminated aerosol [24]. In patients with respiratory failure undergoing NIV via nasal masks, air leakage may occur through the mouth or routes other than the exhalation valve [25]. For example, the patient may loosen the mask strap to relieve discomfort around the nasal bridge, and air leakage from the nasal bridge is definitely a potential source for transmission of viral infection. Careful mask fitting is important for successful, safe application of NIV [24]. Addition of a viral/bacterial filter to the breathing system of NIV between the mask and the exhalation port [13-15] or using dual-circuit NIV may reduce the risk of nosocomial transmission of viral infection [22].

In view of the observation that higher ventilator pressures result in wider dispersion of exhaled air and a higher concentration of air leakage [22, 23], it is advisable to start NIV with a low IPAP level $\left(8-10 \mathrm{cmH}_{2} \mathrm{O}\right)$, gradually increasing it as necessary. Indeed, SARS-related ARF has been reported to respond readily to low positive pressures with CPAP $\left(4-10 \mathrm{cmH}_{2} \mathrm{O}\right)$, IPAP $\left(<10 \mathrm{cmH}_{2} \mathrm{O}\right)$, and EPAP of $\left(4-6 \mathrm{cmH}_{2} \mathrm{O}\right)$ [26]. Higher pressures should be avoided because of the common findings of spontaneous pneumomediastinum and pneumothorax in SARS [1, 10, 12].

During the 2009 pandemic of influenza A(H1N1)pdm09 infections, it was recommended that NIV be applied to suitable patients (e.g., those with mild to moderate acute hypercapnic respiratory failure or acute pulmonary edema or those with resolving ARDS) via a helmet mask with double-circuit tubes or a total fullface mask with filters and avoidance of heated humidification. This was in addition to prudent isolation of the patients coupled with protective measures for the HCWs and other patients (Table 13.2) [27]. The Health Protection Agency, UK, has recommended airborne precaution when applying NIV in patients with the 
H1N1pdm09 infection [28]. Also, the most recent World Health Organization (WHO) interim guidance on management of the novel $\mathrm{CoV}$ has also recommended the use of NIV for mild cases of ARDS without hemodynamic instability [29].

The WHO interim guidelines on prevention and control of acute respiratory diseases in health care has included NIV among those aerosol-generating procedures in which there is possibly increased risk of respiratory pathogen transmission. In addition to maintaining contact, droplet, and standard precautions among HCWs when providing routine care to such patients, the WHO recommends full PPE for the HCW, covering the torso, arms, eyes, nose, and mouth. It includes a long-sleeved gown, single-use gloves, eye protection, and an N95 mask or equivalent as the minimum level of respiratory protection. NIV should be provided in an adequately ventilated single room. There should also be an expiratory port with a bacterial/viral filter that reduces aerosol emission [30].

\subsection{Future Research}

Emerging infectious diseases such as SARS and H1N1pdm09 are highly infectious and are associated with significant morbidity and mortality. NIV may play a limited supportive role for early ARDS/acute lung injury as a bridge to invasive mechanical ventilation in SARS and other emerging respiratory infections. It is contraindicated, however, in critically ill patients with multi-organ failure and hemodynamic instability $[24,27,29]$. As the application of NIV may potentially disperse infected aerosols [22, 23], further research is needed to examine the safety and exhaled air dispersion distances during application of NIV via mask, including the helmet, using double-circuit tubing. When we have a better understanding of these areas, HCWs can better protect themselves within the dangerous distances when managing patients with ARF due to highly infectious diseases. More research is also needed regarding technical improvements of the NIV masks and viral/bacterial filters, as well as in the design of a safer hospital ward environment, to prevent nosocomial transmission of these infections. Advances in knowledge in these research areas can facilitate management of ARF due to future SARS outbreaks and other emerging infectious diseases, such as pandemic influenza.

\section{Key Major Recommendations}

- NIV may play a supportive role for early ARDS/acute lung injury as a bridge to invasive mechanical ventilation in patients with SARS and other emerging infections, although it is contraindicated in critically ill patients with multi-organ failure and hemodynamic instability.

- In addition to strict contact and droplet precautions, HCWs should have adequate respiratory protection when managing patients with SARS as the application of NIV may disperse potentially infected aerosols. 
- Further research is needed to determine the exhaled air dispersion distances and safety during application of NIV via masks (e.g., helmet masks, total full-face mask) and the use of dual circuits so health care providers can better protect themselves within dangerous areas when managing patients with ARF due to highly infectious diseases.

- More research is needed regarding technical improvements in noninvasive positive-pressure ventilation masks, viral/bacterial filters, and the design of safer hospital ward environments with adequate air changes to prevent nosocomial transmission of these infections.

\section{References}

1. Lee N, Hui DS, Wu A, et al. A major outbreak of severe acute respiratory syndrome in Hong Kong. N Engl J Med. 2003;348:1986-94.

2. Hsu LY, Lee CC, Green JA, et al. Severe acute respiratory syndrome in Singapore: clinical features of index patient and initial contacts. Emerg Infect Dis. 2003;9:713-7.

3. Booth CM, Matukas LM, Tomlinson GA, et al. Clinical features and short-term outcomes of 144 patients with SARS in the greater Toronto area. JAMA. 2003;289:2801-9.

4. Hui DS, Sung JJ. Editorial: severe acute respiratory syndrome. Chest. 2003;124:12-5.

5. WHO. Summary of probable SARS cases with onset of illness from 1 November to 31 July 2003. Available at: http://www.who.int/csr/sars/country/table2004_04_21/en/index.html.

6. Peiris JS, Lai ST, Poon LL, et al. Coronavirus as a possible cause of severe acute respiratory syndrome. Lancet. 2003;361:1319-25.

7. Lau SK, Woo PC, Li KS, et al. Severe acute respiratory syndrome coronavirus-like virus in Chinese horseshoe bats. Proc Natl Acad Sci. 2005;102:14040-5.

8. Li W, Shi Z, Yu M, et al. Bats are natural reservoirs of SARS-like coronaviruses. Science. 2005;310:676-9.

9. Peiris JS, Chu CM, Cheng VC, et al. Clinical progression and viral load in a community outbreak of coronavirus-associated SARS pneumonia: a prospective study. Lancet. 2003;361: 1767-72.

10. Hui DS, Wong KT, Antonio GE, et al. Severe acute respiratory syndrome (SARS): correlation of clinical outcome and radiological features. Radiology. 2004;233:579-85.

11. Peiris JS, Yuen KY, Osterhaus AD, et al. The severe acute respiratory syndrome. N Engl J Med. 2003;349:2431-41.

12. Hui DS, Wong PC, Wang C. Severe acute respiratory syndrome: clinical features and diagnosis. Respirology. 2003;8:S20-4.

13. Han F, Jiang YY, Zheng JH, et al. Noninvasive positive pressure ventilation treatment for acute respiratory failure in SARS. Sleep Breath. 2004;8:97-106.

14. Cheung TM, Yam LY, So LK, et al. Effectiveness of noninvasive positive pressure ventilation in the treatment of acute respiratory failure in severe acute respiratory syndrome. Chest. 2004;126:845-50.

15. Yam LY, Chan AY, Cheung TM, et al.; Hong Kong Hospital Authority SARS Collaborative Group (HASCOG). Non-invasive versus invasive mechanical ventilation for respiratory failure in severe acute respiratory syndrome. Chin Med J (Engl). 2005;118:1413-21.

16. Xiao Z, Li Y, Chen RC, et al. A retrospective study of 78 patients with severe acute respiratory syndrome. Chin Med J. 2003;116:805-10.

17. Fowler RA, Guest CB, Lapinsky SE, et al. Transmission of severe acute respiratory syndrome during intubation and mechanical ventilation. Am J Respir Crit Care Med. 2004;169: 1198-202. 
18. Raboud J, Shigayeva A, McGeer A, et al. Risk factors for SARS transmission from patients requiring intubation: a multicentre investigation in Toronto, Canada. PLoS One. 2010;5(5): e10717.

19. Yu IT, Xie ZH, Tsoi KK, et al. Why did outbreaks of severe acute respiratory syndrome occur in some hospital wards but not in others? Clin Infect Dis. 2007;44:1017-25.

20. Tran K, Cimon K, Severn M, et al. Aerosol generating procedures and risk of transmission of acute respiratory infections to healthcare workers: a systematic review. PLoS One. 2012;7(4): e35797.

21. Wong BC, Lee N, Li Y, Chan PK, et al. Possible role of aerosol transmission in a hospital outbreak of influenza. Clin Infect Dis. 2010;51:1176-83.

22. Hui DS, Hall SD, Chan MT, et al. Non-invasive positive pressure ventilation: an experimental model to assess air and particle dispersion. Chest. 2006;130:730-40.

23. Hui DS, Chow BK, Ng SS, et al. Exhaled air dispersion distances during noninvasive ventilation via different respironics face masks. Chest. 2009;136:998-1005.

24. Hui DS, Sung JJ. Editorial: treatment of severe acute respiratory syndrome. Chest. 2004;126:670-4.

25. Hill NS, Carlisle C, Kramer NR. Effect of a nonrebreathing exhalation valve on long-term nasal ventilation using a bilevel device. Chest. 2002;122:84-91.

26. Yam LY, Chen RC, Zhong NS. SARS: ventilatory and intensive care. Respirology. 2003;8: S31-5.

27. Conti G, Larrsson A, Nava S, et al. On the role of non-invasive ventilation (NIV) to treat patients during the H1N1 influenza pandemic. ERS and ESICM guideline Nov 2009. Available at: http://www.dev.ersnet.org/uploads/Document/63/WEB_CHEMIN_5410_1258624143.pdf.

28. Health Protection Agency, UK. Pandemic (H1N1) influenza: a summary of guidance for infection control in healthcare settings. Available at: http://www.dh.gov.uk/prod_consum_dh/ groups/dh_digitalassets/@dh/@en/@ps/documents/digitalasset/dh_110899.pdf.

29. WHO. Interim guidance document on clinical management of severe acute respiratory infections when novel coronavirus is suspected: what to do and what not to do. Available at: http:// www.who.int/csr/disease/coronavirus_infections/InterimGuidance_ClinicalManagement_ NovelCoronavirus_11Feb13u.pdf.

30. World Health Organization. Infection and control of epidemic- and pandemic-prone acute respiratory diseases in health care. WHO interim guidelines. World Health Organization, 2007. Available at: http://www.who.int/csr/resources/publications/WHO_CD_EPR_2007_6/en/. 